\title{
Medical contrast media as possible tools for SAXS contrast variation
}

\author{
Frank Gabela ${ }^{\mathrm{a}}$, Sylvain Engilberge $\mathrm{a}^{\mathrm{a},}$ Javier Pérez $^{\mathrm{b}}$ and Eric Girard ${ }^{\mathrm{a}}$ \\ aIBS, CEA, CNRS, UGA, 71 avenue des Martyrs, Grenoble, 38000, France. frank.gabel@ibs.fr \\ bSynchrotron SOLEIL, St. Aubain BP 48, Gif-sur-Yvette, 91192, France \\ *Present address: Galway University, Galway, Ireland.
}

Small angle X-ray scattering (SAXS) is increasingly used to extract structural information from a multitude of soft matter and biology systems in aqueous solution, including polymers, detergents, lipids, colloids, proteins and RNA/DNA.

When SAXS data are recorded at multiple contrasts, i.e. at different electron densities of the solvent, the internal electron density profile of solubilized molecular systems can be probed. However, contrast variation SAXS has been limited by the range of electron densities available by conventional agents such as sugars, glycerol and salt, and by the fact that many soft matter and biology systems are modified in their presence.

Here we present a pioneering SAXS contrast variation study on DDM (n-Dodecyl- $\beta$-DMaltopyranoside) micelles by using two highly electron-rich contrast agents from biomedical imaging which belong to the families of gadolinium-based and iodinated molecules. The two agents, Gd-HPDO3A and iohexol, allowed to attain modifications of the solvent electron density which are 50 to $100 \%$ higher than those obtained for sucrose, and are located between the electron densities of proteins and RNA/DNA. In the case of Gd-HPDO3A, an analysis of the internal micellar structure was possible and compared to results obtained with sucrose.

Medical contrast agents therefore represent a promising class of molecules for SAXS contrast variation experiments. We will discuss potential applications to soft matter and biology systems, including membrane proteins and protein-RNA/DNA complexes, and the complementarity with solvent contrast variation and deuteration in small angle neutron scattering (SANS).

\section{References}

[1] Gabel, F., Engilberge, S., Pérez, J. \& Girard, E. (2019). IUCrJ, in press. 